\title{
Photoionization, line emission diagnostics
}

\section{Lisa Kewley}

\author{
Institute for Astronomy (IFA), University of Hawaii, USA \\ Email: kewley@IfA.Hawaii.Edu
}

\begin{abstract}
Photoionization models for AGN, including Seyfert and LINERs are discussed. These photoionization models can be used to derive emission-line diagnostics for AGN that can determine the properties of the AGN and surrounding ISM, including the relative AGN contribution to the EUV radiation field, the hardness of the AGN radiation field, the ionization state of the gas, and the metallicity of the narrow-line region. It is shown how the AGN emission-line diagnostics are expected to change with redshift. Finally, latest application of these models by the author to wide integral field spectroscopy to separate starburst and AGN contributions in composite galaxies are presented.
\end{abstract}

